\title{
Theory and Practical Steps to Introducing a New 3D Public Health Indicator to Replace BMI Using Existing Population-Based Multidimensional Reference Measurement Sets
}

\author{
David B. STEFAN ${ }^{1}$, Stephen D. WOHLGEMUTH ${ }^{2,3}$, David A. GILBERT ${ }^{4}$ \\ ${ }^{1}$ Novaptus Systems, Inc. Chesapeake, VA, USA; \\ ${ }^{2}$ Eastern Virginia Medical School, Norfolk, VA, USA; \\ ${ }^{3}$ Sentara Comprehensive Weight Loss Solutions, Norfolk, VA, USA; \\ ${ }^{4}$ The Hague Plastic and Cosmetic Surgery Center, Norfolk, VA, USA
}

http://dx.doi.org/10.15221/14.299

Body Mass Index is an aging public health indicator obesity measurement, based solely on height and weight. Despite its shortcomings, it has gained acceptance over time primarily because of its simplicity, and it has been applied not only to individuals, but also to various population groups. Calls to replace BMI with a more modern indicator within the medical community have been growing louder, but the medical community moves very slow and acceptance of a multidimensional health indicator appears to most to be far off. However, by using multinational 3D sizing studies done over the years by the clothing industry, the introduction of a 3D public health indicator as well as a multidimensional population measurement reference set can be implemented in the very near future.

\section{Introduction}

Walk into any clothing store. The section you will probably gravitate towards would be the clothing racks or shelves that display your "size" of clothes, in the fashion that may interest you. If you happen to be fortunate enough to get customer service, the associate might ask you if you know your size. When we think about size in this context we inevitably think in terms of common measurements such as waist, chest or bust circumferences. Or we might think about the standard size clothing that fit us well in the past. A size 42 sport coat, say, and a shirt collar of 16 inches with a sleeve length of 30 inches. A woman might remember that a Size 4 blouse and a Size 6 skirt from a particular clothing line fit her best. We might even think of "extra-small, small, medium, large or extra-large" in terms of casual wear shirt sizes. Though the clothing sizes differ regionally and measurements may be expressed in units of centimeters or inches, this conditioned way of thought is repeated almost anywhere by clothes shoppers around our globe.

Most of us can't help that. We have been invariably trained since birth to think about clothes in terms of uniform "sizes" and common measurements. We don't stop for a moment and think about how those clothing sizes were developed, let alone the technology behind that development.

But what if I walked into a clothing store, and if asked by the associate whether I knew what my "size" was I answered like this, "I have a torso height of $65 \mathrm{~cm}$. My torso surface area is 6130 squared centimeters, and I have a torso volume of 57,600 cubic centimeters". You undoubtedly would get a blank stare from the associate. Yet what I said would certainly be true. I do have those dimensions. And that is my "size". I know, because I have scanned myself in a 3D body scanner.

What if I would have handed the associate a memory chip containing my 3D scan image exported as a data modeling computer file? Such a file would contain thousands of points that collectively represent my physical outline, with each point having a unique Cartesian coordinate on the surface of my body, as measured from the center of the floor of the scanner. This, in turn, would certainly be an answer to the question, "Do you know your size?"

These examples are mentioned to highlight how confined and regimented our thinking can become, both in the clothing world and in the medical world. Both worlds often look past each other, not realizing how closely linked they really are. The medical world has no real clue about the technology advances that have taken place over the last decade within the clothing world, and how vast sets of data that have been collected on behalf of the clothing industry for clothes sizing purposes can be used to solve a problem that has been vexing public health officials for some time.

That problem is how to update an outdated obesity health indicator, Body Mass Index (BMI) which is based on height and weight, with a modern, multidimensional obesity indicator without taking years to collect, process and analyze data. 
This paper contends that such a leap is indeed quite possible, and practical as well. Most of the "heavy-lifting" has already been done by the clothing world in the form of 3D clothing reference models developed from the extensive clothes sizing studies undertaken by the clothing industry. Adopting the $3 \mathrm{D}$ clothing body model references used to produce apparel for geographically diverse populations can also yield a multidimensional measurement reference set suitable for public health monitoring purposes. The hard and tedious efforts of data collection, de-identification and normalization have already been accomplished. What remains is for the medical community to accept this data, and format and process measurement information in such a way that the medical community can understand and use.

A simple formula, based on measurement components that already exist in all the scans of all the individuals which form the $3 \mathrm{D}$ clothing reference body models has been developed. Acceptance of which could set the stage for a rapid transition from the older world of height and weight, to a new, multidimensional world incorporating surface area and volume. It is a small step towards this new world, but once the transition has been accomplished, vast amounts of new information regarding the physical measurements of the human body can be made available for collaborative research.

\section{Background}

There are two historical tracks that brought us to a point of convergence. One is the medical path, the other is clothing. Both will be examined.

But first, an understanding of what a "Public Health Indicator" is, and in particular what role the Body Mass Index (BMI) plays as a measurement of a public health indicator will be helpful.

One definition of a public health indicator is: "A characteristic of an individual, population or an environment which can be measured and used to describe one or more aspects of the health of an individual or population"'.

There are a number of public health indicators, from tobacco usage and substance abuse to injury and violence. The United States has selected 12 public health indicators and has designated them to be "Leading Health Indicators"2. Figure 1 depicts these Leading Health Indicators. Each one of these Leading Health Indicators has one or more measurements that are used in some way to quantify its condition.

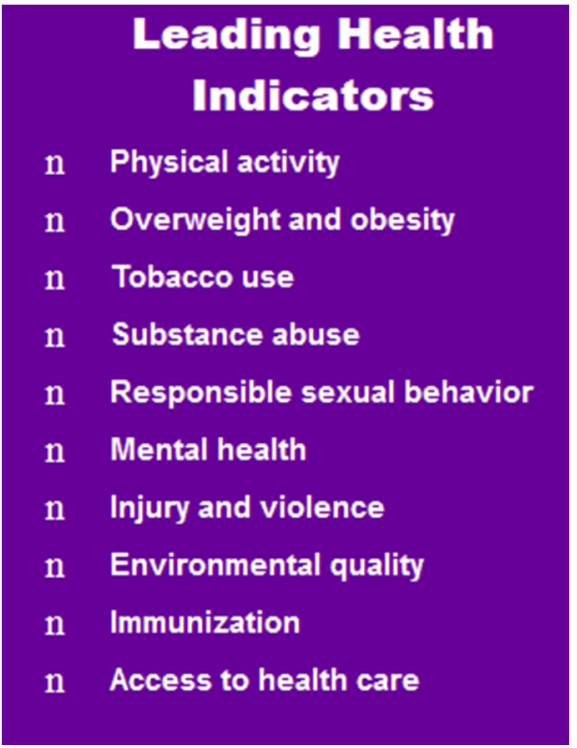

Figure 1. Leading Health Indicators as Selected by the United States Government

The level of obesity in a population is an example of a public health indicator. BMI is a familiar method of measuring obesity. The principal components of BMI are height and weight. In the metric system, it is expressed in terms of $\mathrm{kg} / \mathrm{m}^{2}$. The base components each have one dimension. The height component in the denominator may be squared, but it is not a measurement of surface area. 
BMI is a simple obesity measurement. It was developed by Belgian scientist Adolphe Quetelet in the 1830 s and was known as "Quetelet's Index"3. The name "Body Mass Index" and its subsequent popularity came from a paper published in the July 1972 Journal of Chronic Diseases by Ancel Keys ${ }^{4}$. BMI was explicitly cited by Keys as being appropriate for population studies. Nevertheless it has become widely used as an individual obesity indicator. All that is needed is a scale and a stadiometer to get the component measurements. This simplicity is also its weakness. When applied at the individual level, it penalizes shorter people and those with a muscular framework, and it doesn't differentiate between the morbidly obese and the super obese, or the "healthy" thin and dangerously anorexic.

One criteria to be considered a candidate for surgical weight loss is to have a BMI of 40 or greater ${ }^{5}$. It is not uncommon for a particular class of athletes to have such a BMI number. Blindly looking at a subject's BMI number would lead to a false indication of obesity for such individuals.

The BMI scale for the United States in displayed in Figure 2 below ${ }^{6}$.

\begin{tabular}{|l|l|}
\hline \multicolumn{1}{|c|}{ Category } & BMI range $\mathbf{~} \mathbf{k g} / \mathbf{m}^{2}$ \\
\hline Very severely underweight & less than 15 \\
\hline Severely underweight & from 15.0 to 16.0 \\
\hline Underweight & from 16.0 to 18.5 \\
\hline Normal (healthy weight) & from 18.5 to 25 \\
\hline Overweight & from 25 to 30 \\
\hline Obese Class I (Moderately obese) & from 30 to 35 \\
\hline Obese Class II (Severely obese) & from 35 to 40 \\
\hline Obese Class III (Very severely obese) & over 40 \\
\hline
\end{tabular}

Figure 2. BMI Scale for the United States

BMI categorization is not uniform. It can vary among different populations. The Hospital Authority of Hong Kong recommends the guidelines displayed in Figure $3^{7}$.

\begin{tabular}{|l|l|}
\hline \multicolumn{1}{|c|}{ Category } & BMI range $-\mathbf{~ k g} / \mathbf{m}^{2}$ \\
\hline Underweight & $<18.5$ \\
\hline Normal Range & $18.5-22.9$ \\
\hline Overweight - At Risk & $23.0-24.9$ \\
\hline Overweight - Moderately Obese & $25.0-29.9$ \\
\hline Overweight - Severely Obese & $\geq 30.0$ \\
\hline
\end{tabular}

Figure 3. BMI Scale for Residents of Hong Kong

There are other regional guidelines as well.

There is growing awareness within the medical community regarding the usefulness of BMI as a measurement for a health indicator. In a paper published in August 2006 medical journal Lancet, the researchers looked at 40 studies involving 250,000 patients. Their analysis revealed that people with a BMI of $30-35$ were at lower risk of cardiovascular disease than those whose BMI was below $20^{8}$.

This realization had led to attempts to revise the basic BMI formula. There have been efforts to adopt "BMI Prime"," which is a simple modification, as well as "BMI-for-age"10 which allows comparison between children of the same sex and age ${ }^{11}$. There have also been attempts to supplement BMI with other ratios such as waist-to-hips, or even just the circumferential waist measurement ${ }^{11}$. 
All this seems to be missing an important point. There is a limitation to any obesity indicator if it is just tethered to height and weight, or circumferential measurements such as waist or hips. The medical community is slow to adapt to new ideas. It lurches along, trying to find consensus based on rigorous and methodical data collection and analysis. It has strict protocols about data collection, deidentification and normalization. The end result is that it takes many years to adopt a new method of measuring a public health indicator. This is part inertia and part caution, but it also could be because of the regimentation in the general thought process spoken of earlier, where the belief is that solutions to such problems can only be found within the medical community itself.

\section{Medical background}

As a whole, 3D scanning in the medical world is not new. It has been around for quite some time. Devices employing CAT or MRI produce 3D images. Though these machines can measure surface contours, their use is primarily to investigate internal issues within the body. 3D body scanning to specifically measure a subject for anthropometric data collection is a relatively recent development.

In 2002, such scanning began with plastic surgery patients at a clinic in Norfolk, Virginia. The early 3D body scanners had a prohibitively large footprint and were relatively expensive. The scanner could only fit in an open storage area, not a typical medical examination room. Now, 3D body scanners come in smaller dimensions, costs have come down, and economical handheld scanners are on their way.

The purpose was to investigate the measurement capabilities of the scanning device's software, and to see if these measurements could lend new insight on how to measure and evaluate preoperative and postoperative body contours. The machine was referred to not as a 3D body scanner, but as a "3D Digital Camera", capable of viewing the subject at various angles. Of particular interest was the software's ability to extract surface area and volume measurements.

A measurement template was constructed to document preoperative and postoperative breast measurements. This was applied to subjects who underwent a breast augmentation surgical procedure. The measurement template included linear and circumferential measurements of interest, as well as surface area and volume calculations. Measuring changes to the breasts required measuring changes to whatever the scanner determined to be the subject's torso. Fortunately, the scanner software was fairly consistent in defining the subject's torso for both preoperative and postoperative scans, so changes to the breasts in terms of volume and surface area could be inferred by measuring changes to the subject's preoperative and postoperative torso volume and torso surface area.

In late 2005 we began scanning preoperative bariatric patients to document their physical measurements. These patients then came back for their postoperative scans. We did not weigh these patients at the time of either scan, though their weight loss progress was visually evident. The same technique of comparing measurements of the preoperative torso to the postoperative torso was employed to get a determination of volume and surface area changes.

These early torso measurements techniques were used on two completely different sets of subjects and two completely different surgical procedures. The breast augmentation procedure added volume and surface area, surgical weight loss reduced volume and surface area.

The only item that these subjects had in common was torso height. All individuals have a torso height, and that torso height is usually fixed for the adult individual. The torso height is also range bound over the adult population.

The scanner software determined the torso height from the back of the neck point (neck_back_center point) to the "crotch point", or the beginning of the separation between the torso and the left and right legs. This definition of the torso included the shoulders, but not the head. It also included the buttocks and the hips. Because of these inclusions, it was felt that the torso could act as a reasonable proxy for the entire body. Figure 4 depicts how the scanner software defines the torso of a subject, and how torso surface area and torso volume are calculated. 

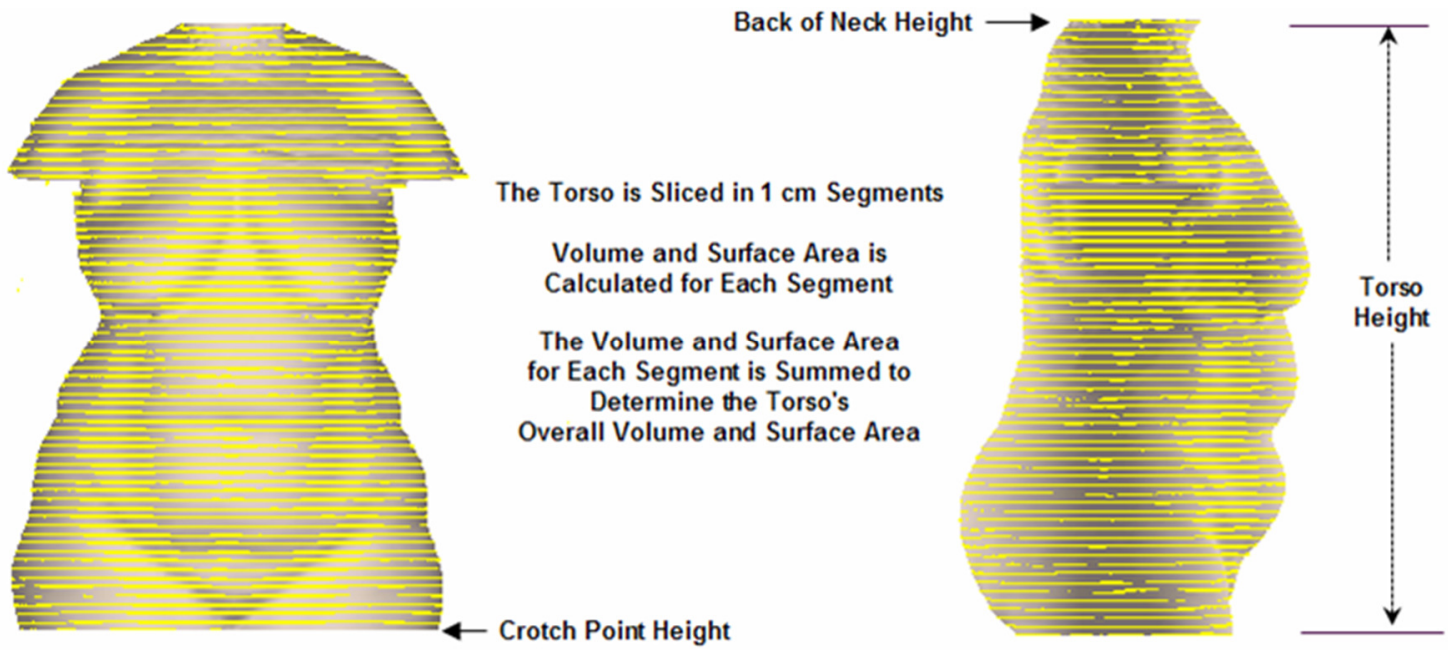

Figure 4. The Scanner Software Definition of the Scanned Subject's Torso

Because the torso height is fixed for an adult, it can be held constant over time. Longitudinal changes to an individual subject's torso volume and torso surface area could then be examined. These could be compared on a relative basis to other individuals. Since the torso height is range bound, individuals that have the same torso height could also be directly compared to one another.

Measurement comparisons were made of the torso volume and torso surface areas of morbidly obese, obese and progressively thinner individuals who had the same torso height. It was discovered that there was a relationship between torso volume and torso surface area, given a fixed torso height. The more obese individuals had a higher torso volume relative to their surface area. Thinner individuals had a higher torso surface area relative to their torso volume.

A good analogy would be to think of a single sheet of paper. This sheet of paper has a large surface area (front and back) relative to its volume (a single sheet thickness). It could be considered "thin". As you add paper to form a stack, the volume begins to increase at a faster rate than the surface area. It starts getting "fatter".

Dividing the torso volume by the torso surface area produced a ratio called the Torso Volume/Surface Area ratio, or TVSA. For subjects with the same torso height, individuals who were more obese had a higher TVSA than individuals who appeared thinner. Figure 5 displays three torsos that have the same torso height. The ectomorph torso has a lower TVSA than the endomorph torso, which in turn has a lower TVSA than the morbidly obese torso.

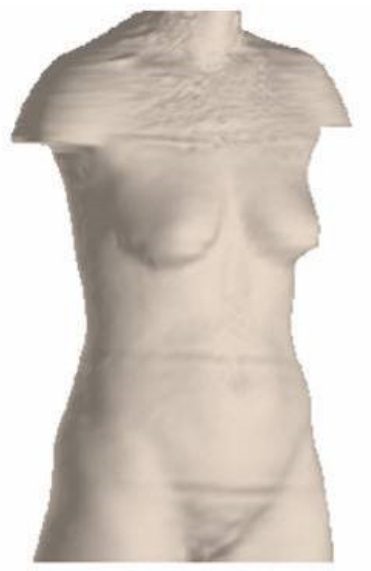

Ectomorph

Torso Volume $31860 \mathrm{cc}$ Torso Surface Area $5391 \mathrm{sq} \mathrm{cm}$ TVSA $=5.91$

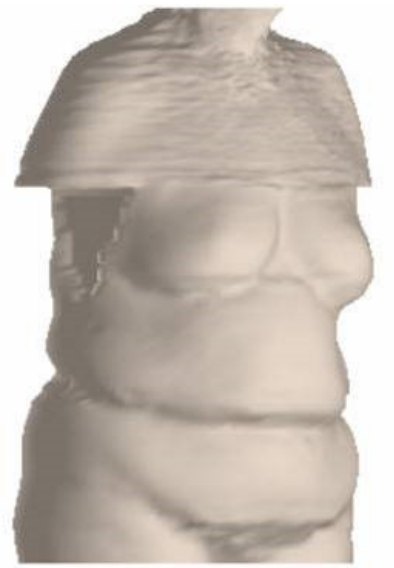

Endomorph

Torso Volume $55921 \mathrm{cc}$ Torso Surface Aroa $7135 \mathrm{sq} \mathrm{cm}$ TVSA $=7.84$

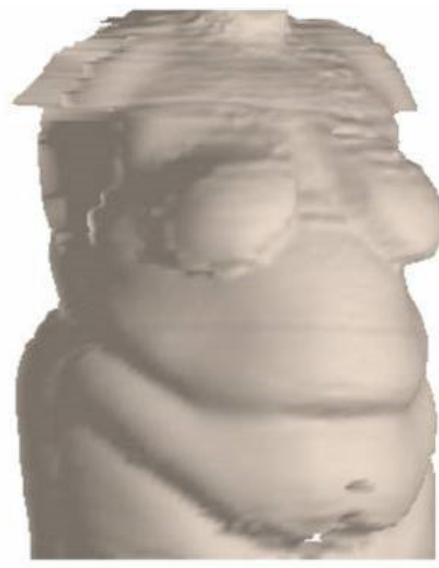

Morbidly Obese

Torso Volume $84858 \mathrm{cc}$ Torso Surface Area $8667 \mathrm{sq} \mathrm{cm}$ TVSA $=9.79$

Figure 5. TVSA Calculations Show Relationship between Torso Volume and Torso Surface Area when Torso Height is Fixed $(64 \mathrm{~cm})$ 
One illuminating example of determining the "space" an individual occupies using TVSA versus the subject's height and weight is depicted in Figure 6. There are two subjects, and each subject has the same height and weight. Since they have the same height and weight, they also have the same BMI. The figure on the right is an amateur bodybuilder as reflected by his muscular build. Because of the relationship between torso volume and torso surface area when the torso height is held fixed, one can readily see that the subject on the left has greater volume relative to his surface area, and hence a higher TVSA number that that of the amateur bodybuilder. The higher the TVSA number, the more obese the subject.

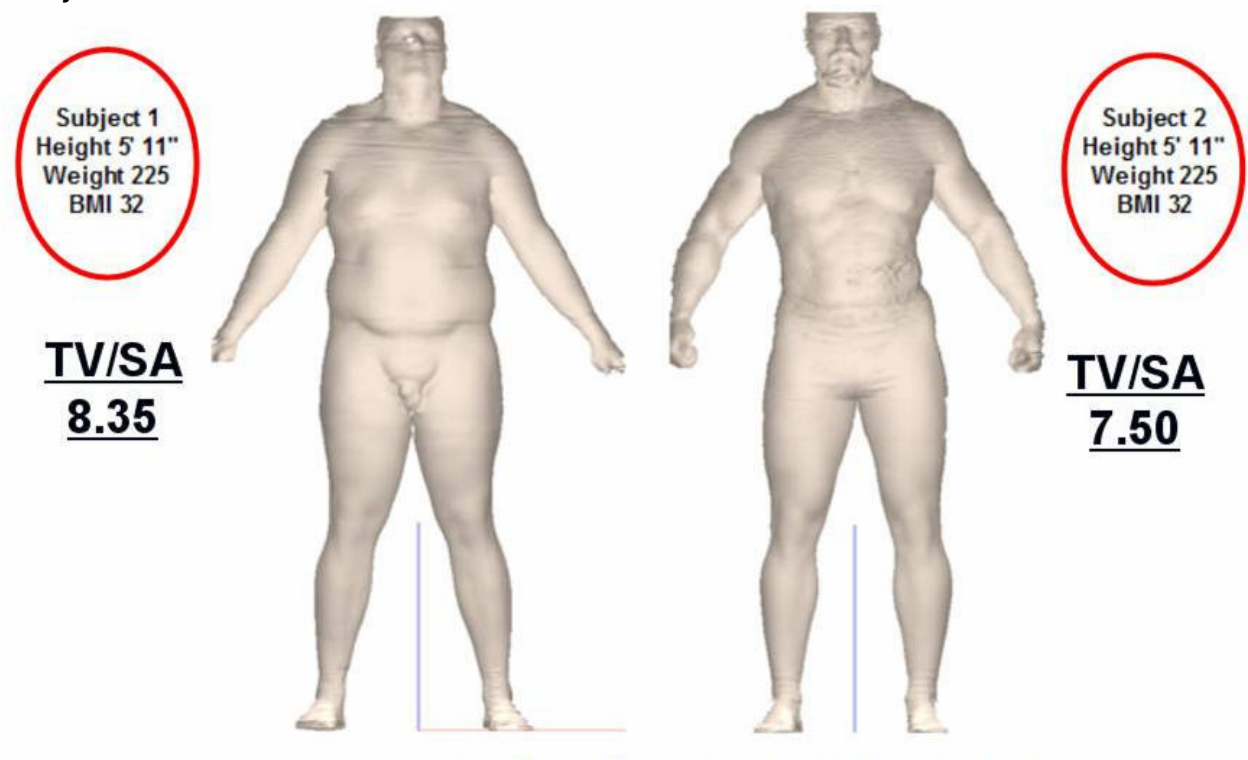

Comparison of Body Shapes Having Same Height, Weight and BMI

Figure 6. The TVSA Measures the "Space" a Subject Occupies

A formula was developed that produced a dimensionless number. This formula used torso height, in $\mathrm{cm}$ in the numerator, divided by the product of torso volume/torso surface area. This dimensionless number has been named the Bariplex.

A scale was developed for interpreting this number. Hundreds of individuals, both male and female, of various shapes, sizes and height were scanned. The formula was applied to this universe of 3D body scans and a statistical analysis was performed.

The scale that resulted from this analysis is displayed in Figure 7. This is called the Generalized Bariplex Scale and is suitable for evaluating both the individual and a general population group. This scale was developed without knowing the weight of any of the individuals. There is no weight component. Instead, this number represents the "normalized space" that someone occupies. It is a different measure of obesity that consists of multidimensional measurement components. These components are common to everyone, but could not readily be obtained without the 3D body models provided by the scanning system and the means to extract volume and surface area measurements.

\begin{tabular}{|c|c|c|}
\hline$\%$ & BariPlex & Indication \\
\hline $91-100$ & 12.596 & Problematically Thin \\
\hline $50-90$ & 11.345 & Proportionally Ideal to Thin \\
\hline Less than 50 & 9.814 & Slightly Overweight \\
\hline Less than 40 & 9.470 & Overweight \\
\hline Less than 30 & 8.944 & Obese \\
\hline Less than 20 & 8.518 & Extremely Obese \\
\hline Less than 10 & 7.783 & Morbidly Obese \\
\hline
\end{tabular}

Figure 7. The Generalized BariPlex Scale 
A subject who has a Bariplex value between 11.345 and 9.815 is considered proportionally ideal to thin. The Generalized Bariplex scale was constructed to emphasize subjects that have varying degrees of obesity.

Specific Bariplex scales can be created that are of particular interest to a medical discipline. Since we have scanned so many preoperative morbidly obese subjects, a specialized Bariplex scale has been developed. It is called the Bariatric Index. This scale differentiates between the super-obese and the morbidly obese and tracks the changes to subjects' torso volume and surface area as they lose weight as a result of a surgical weight loss procedure. Monitoring changes to a bariatric subject's Bariplex and TVSA is an effective method to monitor weight loss progress, even though these measurements do not have a weight component. As bariatric patients lose weight, the amount of "space" they occupy is reduced accordingly. The multidimensional components that comprise TVSA and the Bariplex measure this "space".

\section{Clothing background}

The clothing world is often thought of as a "low tech" industry, replete with images of rows of workers using sewing machines in a cavernous facility located somewhere in a developing country. This is a misperception. Another misperception is that the 3D body scanner is best used for custom-tailored clothing, it is not.

The clothing industry today is a modern marvel, especially when it comes to clothes sizing. This is where 3D body scanning gained early acceptance. Clothing sizes are standards that clothing manufacturers agree to adhere to (for the most part) in order to produce "ready to wear" clothes for the mass market. Clothes sizes used to be based on manikins, and before that, simple tape measurements submitted on punch cards and sent to mail order catalog clothing manufacturers. Now, they are based on 3D reference body models. These 3D clothing reference body models are composed of hundreds and sometimes thousands of individual scans collected from numerous global clothes sizing studies. These scans are processed, analyzed and then normalized into clothes size categories. Figure 8 indicates clothing studies that have been conducted around the world. Many of these are still ongoing. Some are just getting started.

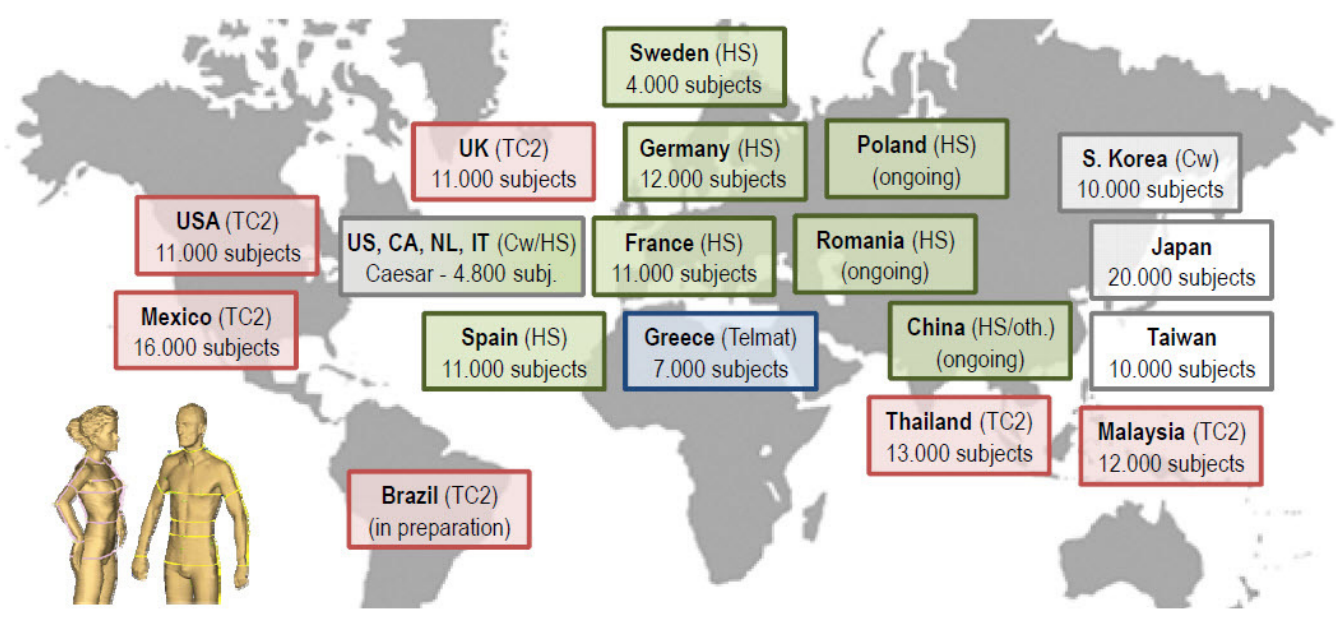

Figure 8. Sample List of Past and Active Clothes Sizing Studies (Figure courtesy of Human Solutions of North America, Carey, NC. USA)

The result of these clothing studies are standardized 3D clothing reference body models. They look like 3D manikins because of the required scanning position. Each series of 3D clothing reference models have a particular height and sometimes a particular shape. They vary in "girth" so that women that are, say 5' 4", have an array of clothes sizes to choose from that are designed for their approximate height.

Figure 9 depicts a series of "Plus Size" 3D clothing reference body models turned into manikins. Note that they have the same height. However, each of these 3D clothing reference models have different circumferential measurements such as hips, bust, waist and thighs. 

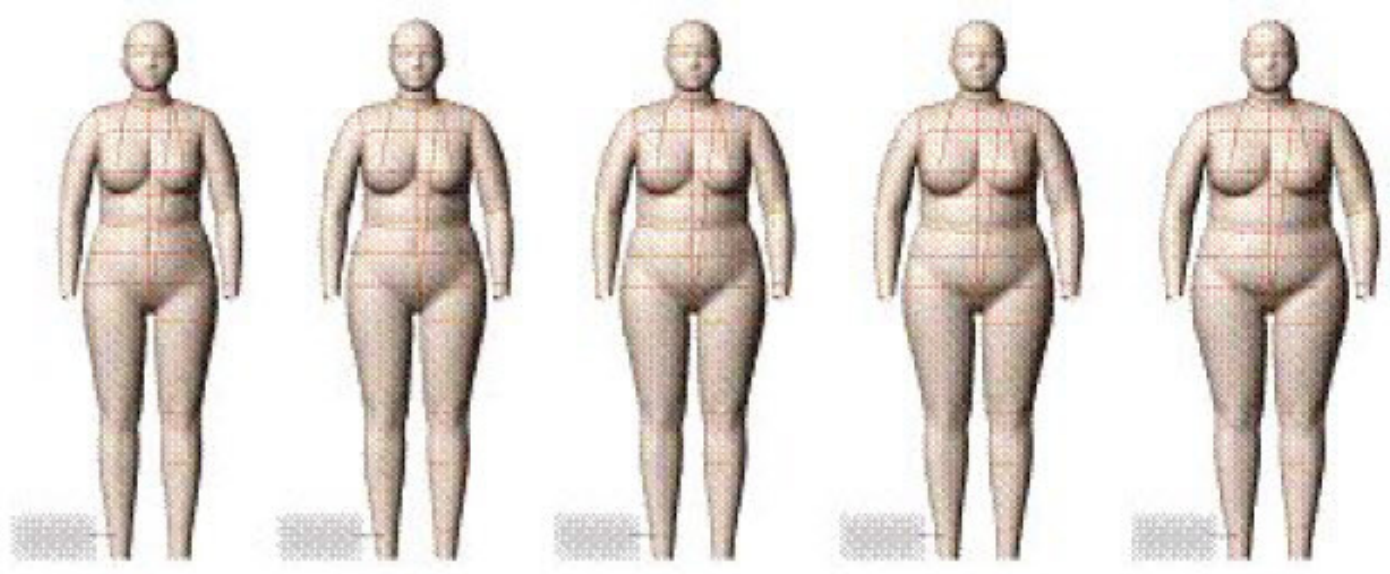

Figure 9. "Plus Size" Manikins Created from 3D Clothing Reference Body Models (Figure courtesy of Alvanon, Inc.)

This is a global gold mine of information, just waiting to be used by the medical community, and in particular public health officials on a country or even worldwide basis.

How so? Think of a 3D body model as nothing but a "container" full of potential measurements. This container can be unlocked. What one gets out of this container depends on the measurements one wishes to extract. The clothing world generally uses 1D measures, such as waist, bust, hips, arm and leg length as part of the process of developing a cutting pattern for ready to wear clothing. It often disregards the multidimensional surface area and volume information that exists within the 3D body model.

A common example of how 1D information is used within the clothing industry would be the construction of a shirt or a pair of pants. The many separate pieces of fabric required to construct a shirt or a pair of pants were created by measuring a 3D body reference model, or even a body model of an individual in the case of custom-fitted clothes, in a particular way. All these individual fabric pieces are then "flattened" for sewing purposes and can be expressed with a single dimensional unit (inches or centimeters). When these pieces of fabric are sewn together, they become a multidimensional object.

Figure 10 shows an example of a subject's 3D body model sized for a pair of pants and a vest. The clothes pattern is "flattened", thereby removing any multidimensional measurements.

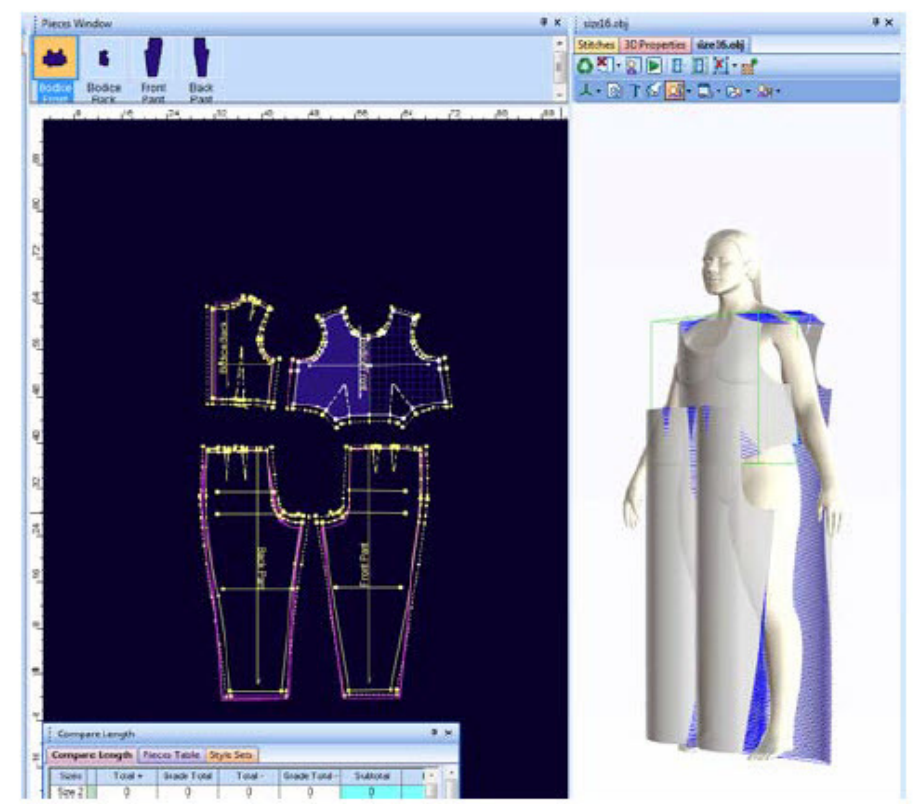

Figure 10. An Example of "Flattening" 3D Scan Information into a 1D Clothes Pattern (Figure courtesy of Optitex, Inc.) 
3D clothing reference body models do not have a weight component. This point may seem obvious, yet it is very subtle, and very powerful. When you pass by a storefront displaying clothes on manikins, have you ever asked yourself, "I wonder how much those manikins weigh?" Probably not. Clothing sizes have no direct linkage to weight. Yet the clothing industry mass produces clothes that fit all body shapes and sizes. Clothing selections range from "Junior Petite" to "Big and Tall", all without regard to the weight of the person who might want to purchase those clothing articles.

The absence of a weight component in the 3D clothing reference body models is the key to making the jump from today's BMI to a multidimensional obesity indicator. The component measurements for the Bariplex; torso height, torso surface area and torso volume exist within all the 3D reference clothing body models, and they exist within all the separate scans of individuals that comprise a particular 3D clothing reference body model.

3D clothes reference body models reflect a composite of geographically diverse males and females that represent a valid cross section of a population. In many ways, they are the population. There could be no other way to satisfy the clothing needs of the public on a mass manufacturing and distribution scale if it was otherwise.

\section{The Theory Summarized}

There exists a relationship between torso volume and torso surface area such that when the torso height is fixed, subjects that are more obese have a greater torso volume/torso surface area number. Subjects that are thinner have a lower torso volume/torso surface area number. This number is called the Torso Volume Torso Surface Area ratio, or TVSA.

There exists a dimensionless number that is found by dividing the torso height with the TVSA ratio. That number is called the Bariplex. A generalized scale has been created to interpret this number based upon statistical analysis of a universe of 3D body scans. The lower this number the more obese the subject.

The TVSA and Bariplex do not have a weight component.

Clothes sizes are based on 3D clothing reference body models. These body models were created by clothes sizing studies using thousands of 3D body scans that represent a cross section of a general or specific population group.

These 3D clothing reference body models are anonymous. The underlying body scan data that comprise a particular 3D clothing reference model has been de-identified. The 3D clothing reference body models are normalized, generally by height.

These 3D clothing reference body models do not have a weight component.

The component measurements for calculating TVSA or the Bariplex exist within all the body models that comprise a particular 3D clothing reference body model, as well as the 3D clothing reference body model itself.

Therefore, applying the Bariplex formula to a particular set of 3D clothing reference body models would be a valid way of introducing a multidimensional method to measure the level of obesity for a particular population. The level of obesity for a particular population being a public health indicator.

\section{Proposed methods}

The question comes down to which set of $3 \mathrm{D}$ clothing reference body models does one want to choose from first? The Bariplex calculation is a measure of obesity valid for all 3D body models, even those that are meant to size clothes for children, though a specialized pediatric index for children has yet to be developed.

Since the Bariplex number scale was created using body scans from American men and women, the SizeUSA clothing size study seems to be the logical place to start. SizeUSA was a clothing study that took place in 2004. The study scanned over 10,000 males and females in 13 cities across the USA. This study mirrored the statistical profile conducted by the Center for Disease Control in one of their National Health and Nutrition Examination Surveys. It is interesting to note that of the 240 measurements taken as part of this study, no analysis was conducted on multidimensional 
measurements. That is, the sponsors of the study as well as the clothing manufacturers who consume the information felt no need to analyze surface area and volume as it pertains to clothes sizing. This certainly is understandable. Fortunately, the 3D body models used for the study are archived and available. As part of the selection process for scan candidates, height, weight and age were recorded. Scan candidates that were especially heavy or extremely light or very short or very tall were not chosen to participate in the study.

Calculating the TVSA and Bariplex for the entire set of SizeUSA 3D body models, or the composite clothing size $3 \mathrm{D}$ reference body models is straightforward. The body models exist in $3 \mathrm{D}$ form. The TVSA and Bariplex can be calculated for each SizeUSA 3D body model or the composite 3D reference clothing models using the batch process capability within the scanning system's software. A measurement profile that determines the torso height, torso surface area and torso volume has already been constructed. Calculations for TVSA and Bariplex can be done within the extraction profile by using reverse polish notation mathematical formulas. The end result of the batch process would be a comma delimited file comprised of all the SizeUSA's de-identified 3D body model names in a column, followed by the columns containing torso height, torso volume, torso surface area, torso volume, TVSA and Bariplex calculations. One could also add the height and weight (hence BMI) as columns in the spreadsheet. Once entered. A full statistical analysis could be done between height, weight, torso volume, torso surface area, and torso height. This would validate the new multidimensional obesity measurements alongside the traditional BMI measurement, facilitating the transition from the old to the new.

The batch process output file can be imported into a spreadsheet and analyzed along with other measurements as indicated in Figure 11.

\begin{tabular}{|c|c|c|c|c|c|c|c|c|c|c|c|}
\hline ID & Weight, Ibs & $\mathrm{kg}$ & Height, In & $\mathrm{cm}$ & BMI & Bulk_Volume_cC & Torso_Height & Torso_Volume_cC & Torso_Surface_Area_with_shoulders & TVSA_Ratio & Bariplex \\
\hline 805A & 348 & 158 & 70 & 178 & 50 & 144371 & 73 & 109899 & 10443 & 10.52 & 6.89 \\
\hline 689B & 303 & 137 & 70 & 178 & 43 & 131058 & 75 & 100765 & 10302 & 9.78 & 7.67 \\
\hline $651 \mathrm{C}$ & 247 & 112 & 67 & 170 & 39 & 106142 & 68 & 76524 & 8572 & 8.93 & 7.56 \\
\hline 265D & 337 & 153 & 70 & 178 & 48 & 143882 & 80 & 111202 & 11035 & 10.08 & 7.89 \\
\hline 654E & 242 & 110 & 69 & 175 & 36 & 101616 & 74 & 79386 & 9133 & 8.69 & 8.54 \\
\hline $789 \mathrm{~F}$ & 260 & 118 & 67 & 170 & 41 & 120165 & 68 & 79775 & 8622 & 9.25 & 7.30 \\
\hline $446 \mathrm{G}$ & 338 & 153 & 73 & 185 & 45 & 142207 & 75 & 103090 & 10346 & 9.96 & 7.48 \\
\hline
\end{tabular}

Figure 11. Example Output of a Batch Process to Extract Volume and Surface Area and to Calculate TVSA and the Bariplex

In this case, the height and weight of the subject was added as spreadsheet columns. Weight information is not available from the scanner, and the scanner system software does a poor job of determining the actual height of the subject (this is often due to dark hair).

The universe of $3 D$ clothing reference body models that comprise SizeUSA can be used to form a multidimensional reference database. Today, the medical community uses simple height and weight charts. Multidimensional information hasn't been available. These charts are generally used for weight management, and are displayed in terms of height, weight and some classification of obesity. Some charts are tabulated by height and recommended weight range based on a particular body frame type. To calculate body frame type one must calculate the circumference of the elbow with the arm held in a particular position. Once calculated, a chart determines the particular body frame category in which you belong. These charts have been developed over years of careful survey, much of which has been provided by insurance company actuarial information. Figure 12 depicts a height weight chart for women. Note that this example chart is categorized by body frame type ${ }^{12}$. 


\begin{tabular}{|l|l|l|l|}
\hline $\begin{array}{l}\text { Height } \\
\text { Feet Inches }\end{array}$ & $\begin{array}{l}\text { Small } \\
\text { Frame }\end{array}$ & $\begin{array}{l}\text { Medium } \\
\text { Frame }\end{array}$ & $\begin{array}{l}\text { Large } \\
\text { Frame }\end{array}$ \\
\hline $4^{\prime} 10^{\prime \prime}$ & $102-111$ & $109-121$ & $118-131$ \\
\hline $4^{\prime} 11^{\prime \prime}$ & $103-113$ & $111-123$ & $120-134$ \\
\hline $5^{\prime} 0^{\prime \prime}$ & $104-115$ & $113-126$ & $122-137$ \\
\hline $5^{\prime} 1^{\prime \prime}$ & $106-118$ & $115-129$ & $125-140$ \\
\hline $5^{\prime} 2^{\prime \prime}$ & $108-121$ & $118-132$ & $128-143$ \\
\hline $5^{\prime} 3^{\prime \prime}$ & $111-124$ & $121-135$ & $131-147$ \\
\hline $5^{\prime} 4^{\prime \prime}$ & $114-127$ & $124-138$ & $134-151$ \\
\hline $5^{\prime} 5^{\prime \prime}$ & $117-130$ & $127-141$ & $137-155$ \\
\hline $5^{\prime} 6^{\prime \prime}$ & $120-133$ & $130-144$ & $140-159$ \\
\hline $5^{\prime} 7^{\prime \prime}$ & $123-136$ & $133-147$ & $143-163$ \\
\hline $5^{\prime} 8^{\prime \prime}$ & $126-139$ & $136-150$ & $146-167$ \\
\hline $5^{\prime} 9^{\prime \prime}$ & $129-142$ & $139-153$ & $149-170$ \\
\hline $5^{\prime} 10^{\prime \prime}$ & $132-145$ & $142-156$ & $152-173$ \\
\hline $5^{\prime} 11^{\prime \prime}$ & $135-148$ & $145-159$ & $155-176$ \\
\hline $6^{\prime} 0^{\prime \prime}$ & $138-151$ & $148-162$ & $158-179$ \\
\hline $\begin{array}{l}\text { Weights at ages 25-59 based on lovest mortality, Weight in pounds according to frame (in indoor dothing veighing 3 lbs.; shoes } \\
\text { vith 1" heels) }\end{array}$ \\
\hline
\end{tabular}

Figure 12. Height Weight Chart for Women (USA)

By creating a measurement profile that consists of linear and circumferential measurements, heights of those measurements, surface area measurements for all body components, and volume measurements, one can produce a true multidimensional reference data base. Such a database could be exploited by comparing the subject's height and weight to all the measurement information extracted from the SizeUSA data set. Instead of height and weight as a measure of obesity and weight management, one could envision comparing TVSA to torso height, or some other combination of measurements not connected to a weight component. A chart depicted in Figure 13 illustrates this concept. The TVSA ratio is plotted along the $y$-axis, and torso height along the $x$-axis. This data was extracted from a series of preoperative bariatric patients. In this case, if the TVSA was above 10, a potential for the subject having a larger liver size than normal might be present, complicating the time it would take for a surgical procedure to be performed.

The scale on the right could be replaced by a descriptive obesity scale, similar to that found for BMI or the Bariplex.

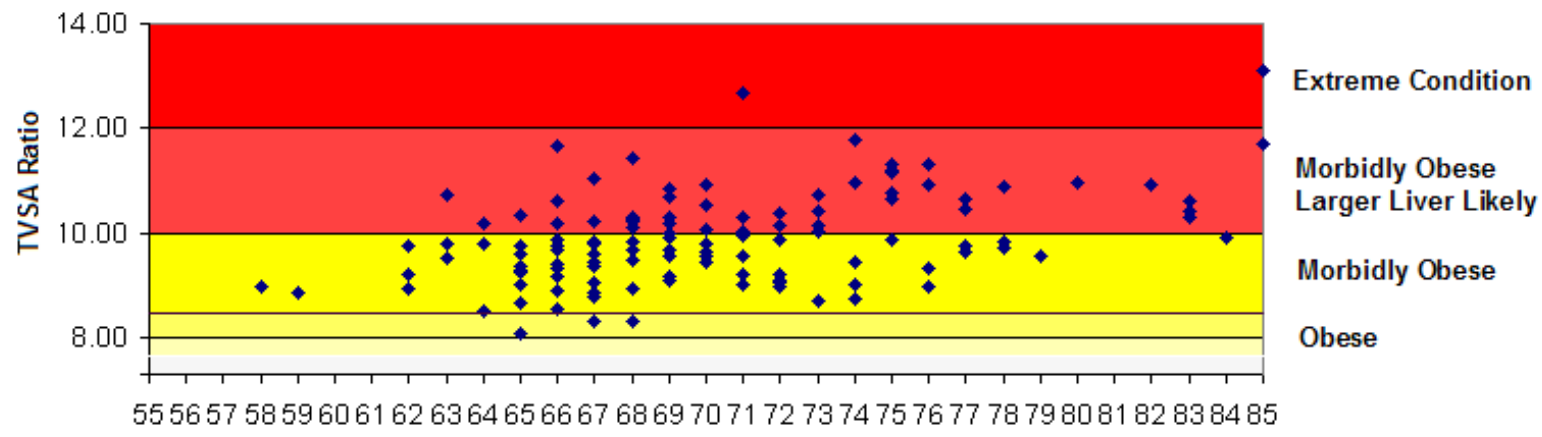

Torso Height, cm

Figure 13. An Example of a Height Surface Area/Volume Chart with no Weight Component

\section{Discussion}

We have seen that it is possible to have a measure of obesity without using a weight component. We have also seen that clothing sizes are based on 3D reference body models and that these reference body models have been developed by aggregating hundreds if not thousands of individual 3D body scans. Clothing sizes reflect the population at large. Thus using 3D clothing reference body models as reference measurements would be a good representation of an adult population. These 3D clothing reference body models do not have a weight component. The individual measurements for the new multidimensional obesity calculation reside within each of the individual body scans that comprise the 
3D clothing reference body models. In fact, these measurements reside in every human. Therefore, it would be valid to assert that calculating these new obesity indicators from the existing 3D clothing reference models would yield an accurate assessment of the level of obesity current among a particular adult population. If this is true, then the TVSA and Bariplex could be accepted and introduced as a measurement of the level of obesity for the population group that was used to create the 3D clothing body model references. There is no need to wait for data collection to occur. It is already there.

In fact, many 3D clothing reference model sets abound as Figure 8 above suggests. These 3D clothing reference body models contain all the measurement information to develop a multidimensional reference data measurement set based on demographics and geography. The models in Figure 14 represent results of a clothes sizing study using 3D scanning to develop 3D clothing reference body models for Chinese men and women at particular ages ${ }^{13}$.
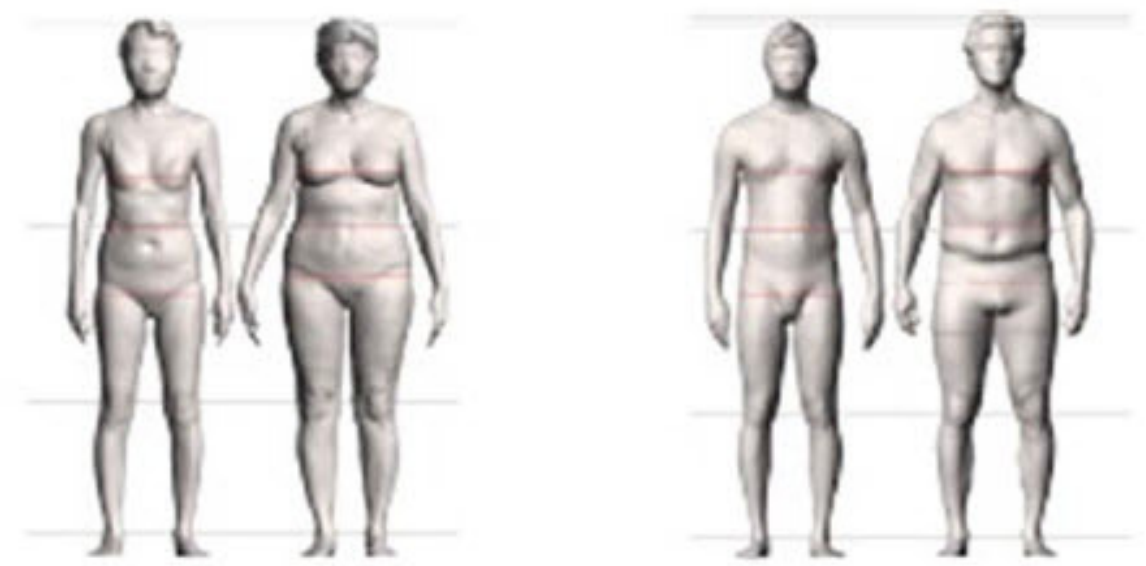

Figure 14. 3D Clothing Reference Body Model Examples from a Chinese Clothing Size Survey (Figure courtesy of Alvanon, Inc.)

A complete statistical analysis can be done on the multidimensional reference data set. This could be compared to the multidimensional reference data set created from the SizeUSA study, or any of the numerous geographical 3D clothes sizing studies that have been conducted. In this way, it might be possible to gain further insight into comparing populations using variables such as surface area and volume rather than just height, weight and circumferential measurements.

There have even been extensive children's clothes sizing studies based on 3D body scanning. Figure 15 depicts a set of manikins derived from 3D clothing reference models created by clothes sizing studies of children.

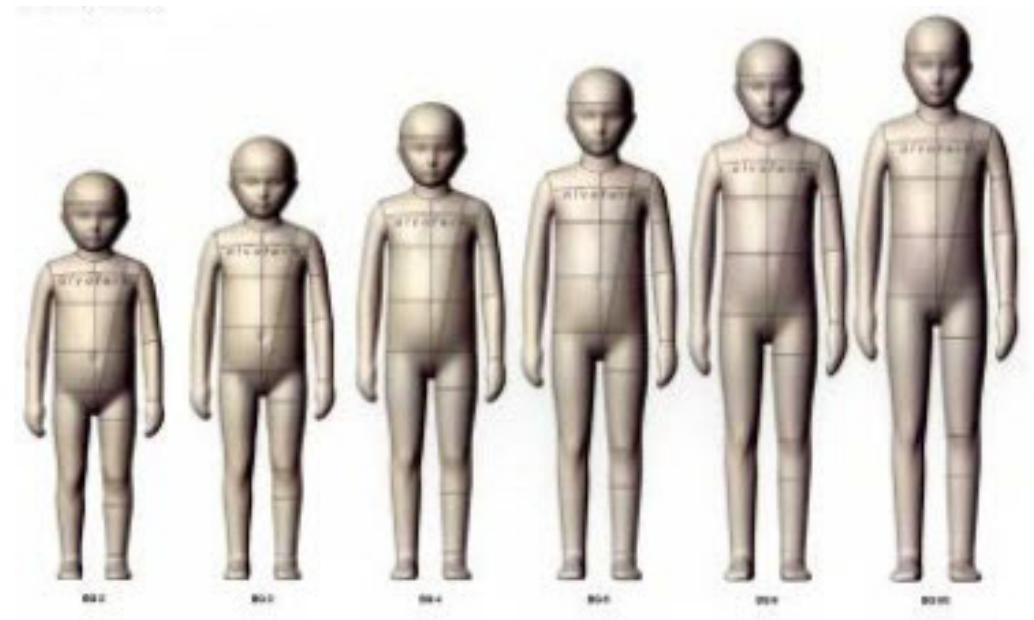

Figure 15. Clothing Reference Body Models for Children (Figure courtesy of ASTM International) 
Instead of the traditional growth charts based on height, weight and age, a new chart could be developed that either replaces or complements the weight component with surface area and volume measurements. This can come directly from the existing 3D children's clothing reference body models and might complement research efforts underway to investigate the onset of juvenile diabetes, as an example.

\section{Conclusion}

One might ask why the introduction of a multidimensional obesity indicator is necessary, and why does it have to be done now? The answer is that 3D body scanning, like other 3D technologies such as $3 \mathrm{D}$ printing are becoming more ubiquitous. It is starting to become part of our daily lives. Soon, you will be able to get a dimensionally accurate $3 \mathrm{D}$ scan from the camera on your game console or a home scanning device, or from the stereoscopic cameras on upcoming smartphones. Economical and easy to operate hand held scanners will become as common as a stethoscope in medical clinics. From such scan sources it will be a fairly straightforward process to measure at least the torso dimensions in 3D. There will be no need for every medical office to have a full body 3D scanning system. Ultimately, all of us will have a dimensionally correct avatar of ourselves which will be useful as we shop for clothing on the web at internet storefronts. This is happening much more rapidly than the medical community realizes. Figure 16 displays an example of a low-cost hand held scanning device that may eventually become just another clinical apparatus.

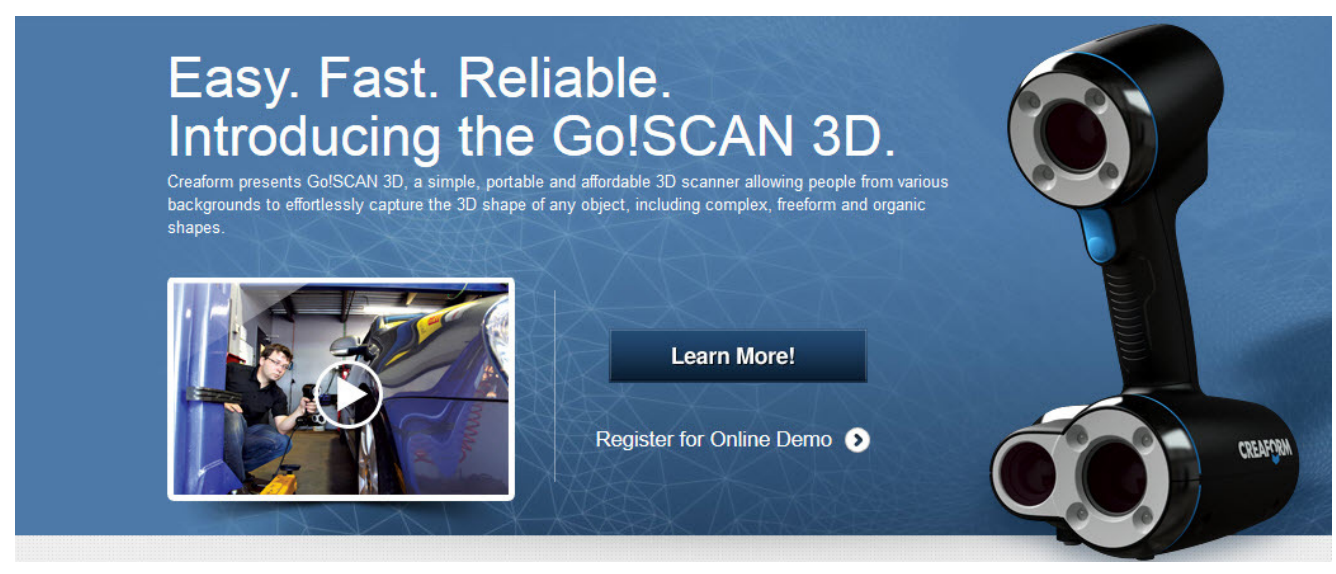

Figure 16. Low Cost and Accurate Hand Held Scanners will become Commonplace

This paper has outlined the theory behind a new type of multidimensional obesity indicator, the Bariplex and a practical method for its immediate implementation. The component measurements that comprise the Bariplex are torso height, torso surface area and torso volume. These measurements exist within all of us and can be gleaned from 3D body models produced by body scanning systems. The Bariplex can be utilized on existing 3D clothing reference body models that collectively represent a particular population. This can at first supplement, then replace BMI as a common measurement of obesity in the public health sense. A transition must eventually be made in the medical world that today is bounded by the use of height and weight, to a new multidimensional world that uses all available body measurements, including surface area and volume. The approach outlined in this paper is a small but crucial step to achieving that goal.

Thanks to the innovations introduced by 3D body scanning and its acceptance and deployment within the clothing world, the foundation for this transition has already been built. The hard work of data collection, de-identification and normalization has already been accomplished. They are in the form of $3 \mathrm{D}$ reference clothing body models. The medical world should accept and incorporate this body of work and build upon it.

The next time you visit a clothing store, look at the variety of apparel and apparel sizes. You will probably find some article of clothing that will fit you, and in a fashion that appeals to you. Now you know a little about the technology used to produce "ready to wear" clothing on a mass basis. And now you know how that same technology and the same 3D body models used for clothes sizing purposes can be used in the medical world.

By the way, just what "size" are you, anyway? 


\section{References}

1. http://definitionofwellness.com/wellness-dictionary/health-indicator/

2. http://www.healthypeople.gov/2010/document/html/uih/uih 4.htm?visit=1

3. Eknoyan, Garabed (2007). "Adolphe Quetelet (1796-1874)- the average man and indices of obesity". Nephrology Dialysis Transplantation 23 (1): 47-51. doi:10.1093/ndt/gfm517. PMID 17890752.

4. Keys, Ancel; Fidanza, Flaminio; Karvonen, Martti J.; Kimura, Noboru; Taylor, Henry L. (1972). "Indices of relative weight and obesity". Journal of Chronic Diseases 25 (6-7): 329-43. doi:10.1016/0021-9681(72)90027-6. PMID 4650929

5. http://consensus.nih.gov/1991/1991 gisurgeryobesity084html.htm

6. "BMI Classification". Global Database on Body Mass Index. World Health Organization. 2006.

7. "BMI正常指標" [Normal BMI Index]. Ideal BMI Disease Prevention Project (in Chinese). Hospital Authority Health InfoWorld.

8. http://www.thelancet.com/journals/lancet/article/PIIS0140673606692519/abstract

9. Gadzik, James (2006). "'How much should I weigh?' Quetelet's equation, upper weight limits, and BMI prime". Connecticut Medicine 70 (2): 81-8. PMID 16768059

10. "Body Mass Index: BMI for Children and Teens". Center for Disease Control.

11. Obesity Index That Better Predict Metabolic Syndrome: Body Mass Index, Waist Circumference, Waist Hip Ratio, or Waist Height Ratio

http://www.ncbi.nlm.nih.gov/pmc/articles/PMC3755383/?report=classic

12. http://www.healthchecksystems.com/heightweightchart.htm

13. http://www.alvanon.com/news/CHINASCAN.pdf 